\title{
La experticia profesional en la judicialización de la violencia doméstica conyugal
}

The professional expertise on the judicialization of Domestic violence against women

\author{
Cecilia Montes Maldonado \\ Universidad de la República
}

Laura López Gallego

Universidad de la República y Universidad Autónoma de Barcelona

\section{Resumen}

El artículo analiza el dispositivo pericial en el campo jurídico en Violencia Doméstica Conyugal ejercida hacia las mujeres. Recoge resultados de una investigación cualitativa con perspectiva etnográfica en el ámbito de los J uzgados de Familia en Uruguay. Nos preguntamos acerca de la construcción de la verdad a través de las cualidades jurídicas y de la evaluación de la personalidad. Discutimos la judicialización como una de las esferas que intervienen sobre la organización de la vida social, la familia y las relaciones de pareja. Analizamos cómo el dispositivo pericial colabora en la construcción de pruebas "confirmatorias", a la vez que legitima ciertos estereotipos de lo que se entiende por violencia, víctima y pareja. La personalidad es pensada desde una lógica individualista, en la que se habilita el pronóstico futuro de comportamientos, dado que existe una unicidad entre acto y actor.

Palabras clave: Violencia doméstica; Género; Dispositivo pericial; Repertorios interpretativos

\section{Abstract}

This paper analyses the expert assemblage inserted in the legal field of Domestic Violence Against Women. It collects results from a qualitative research with an ethnographic perspective in the field of the Specialized Family Court in Uruguay. We inquire about the construction of the truth through the legal qualities and personality assessment as a central element of the expert activity. The discussion deals with the judicialization as one of the various areas involved on the social, family and partner life organization. We analyse how the expert assemblage builds "confirmatory" evidence while legitimates stereotypes of what is meant by violence, victim and couple. The personality is designed from an individualistic logic, which forecast future behaviour is enabled as there is a oneness between act and actor.

Keywords: Domestic Violence; Gender; Expertise Assemblage; Interpretative Repertories 


\section{Introducción. El dispositivo pericial en el campo jurídico de la Violencia Doméstica Conyugal}

En el presente artículo problematizamos el dispositivo pericial inserto en el campo jurídico en Violencia Doméstica Conyugal ejercida hacia las mujeres (VDC). Dicho dispositivo se construye en las intersecciones de las disciplinas "auxiliares" a la J usticia Especializada de Familia -medicina, psiquiatría, psicología y trabajo social-y el derecho de familia en cuestiones de Violencia Doméstica (VD), plasmado en la Ley $\mathrm{N}^{\circ} 17514$.

En este sentido, analizamos un campo de experticia particular, aquel que vincula el saber técnico en el marco de la VDC en los J uzgados Letrados de Primera Instancia de Familia Especializados (J LFE). La noción de experticia (Rose, 1998) que implica el dispositivo pericial, hace referencia a una particular clase de autoridad social, que ejerce una mirada diagnóstica singular. En este caso, aquel que concierne a las concepciones interrelacionadas de violencia, familia, mujer y víctima.

Las prácticas profesionales ligadas al dispositivo pericial constituyen un recorte temático específico, que limita el gran campo de la experticia profesional en el terreno de lo jurídico, que focaliza en lo que se ha dado a llamar actividad pericial. Según lo establecido en el Código General del Proceso (Ley № 15.982), pericia alude a la sabiduría, experiencia o habilidad en una ciencia o arte, mientras que perito es una persona experta en un área específica, que con sus conocimientos asesora en la labor de administración de justicia.

Como forma de realizar un trabajo analítico de la experticia profesional seleccionada, recurrimos a la noción de dispositivo desde las teorizaciones de Michel Foucault, lo que nos lleva a pensar líneas móviles, sin contornos restrictivos, que conforman "cadenas de variables relacionadas entre sí" (Deleuze, 1989/1999, p. 155). En definitiva la apuesta es discutir en términos de prácticas y discursos situados, atravesados por una historicidad que los torna contingentes y cuya apariencia de inmutables es producto de regímenes de verdad a ser tensionados.

Por otra parte, el campo jurídico concerniente a la VDC se encuentra regulado por la Ley №17.514 de VD. Una legislación que pretende alejarse de lo penal expresando un espíritu preventivo, pero que de todas maneras conserva ambigüedades en lo que hace a su capacidad punitiva. Algunos de sus mecanismos coartan y limitan fuertemente libertades personales como son: la disposición del uso de dispositivos electrónicos (tobillera y rastreador) para controlar el cumplimiento de medidas de no acercamiento y la medida de protección que prohíbe o restringe la presencia de la persona agresora del domicilio y otros lugares.

Ahora bien, en lo que refiere a lo específico de la temática elegida, Ia VDC, desde el marco normativo ofrecido por la ley $\mathrm{N}^{\circ} 17.514$ de VD., es definida como:

Toda acción u omisión, directa o indirecta, que por cualquier medio menoscabe, limitando ilegítimamente el libre ejercicio o goce de los derechos humanos de una persona, causada por otra con la cual tenga o haya tenido una relación de noviazgo o con la cual tenga o haya tenido una relación afectiva basada en la cohabitación y originada por parentesco, por matrimonio o por unión de hecho (ley $\mathrm{N}^{\circ} 17.514$, artículo 2).

Dentro de las formas de manifestación de la violencia plantea cuatro tipos; física, psicológica o emocional, sexual y patrimonial. Con respecto a estos tipos definidos, dice Luis $\mathrm{Pa}$ checo (2013) que la misma "es amplísima y tiende a abarcar cualquier tipo de conducta violenta en el seno doméstico-no es taxativa, y se trata simplemente de "manifestaciones" o "expresiones" de la violencia" (p. 28). A su vez las denuncias pueden ser realizadas por cualquier persona, tanto por la persona directamente afectada como por terceras personas. Así se inicia un proceso judicial por medio del cual se pueden disponer medidas de protección judicial para el cuidado de la mujer ${ }^{1}$. Así para la determinación de las medidas

\footnotetext{
${ }^{1}$ Las ocho medidas de protección son: 1 . Disponer el retiro del agresor de la residencia común y la entrega inmediata de sus efectos personales en presencia del Alguacil. 2. Disponer el reintegro al domicilio o residencia de la víctima que hubiere salido del mismo por razones de seguridad personal, en presencia del Alguacil.3. Prohibir, restringir o limitar la presencia del agresor en el domicilio 0 residencia, lugares de trabajo, estudio u otros que frecuente la víctima. 4. Prohibir al agresor comunicarse, relacionarse, entrevistarse o desarrollar cualquier conducta similar en relación con la víctima, demás personas afectadas, testigos o denunciantes del hecho. 5. Incautar las armas que el agresor tuviere en su poder.6. Fijar una obligación alimentaria provisional a favor de la víctima. 7. Disponer la asistencia obligatoria del agresor a programas de rehabilitación.8. Resolver provisoriamente
} 
y la toma de decisiones en el proceso judicial es que se solicita asesoramiento al equipo técnico (ETEC).

En este marco normativo, el ETEC, es decir la experticia profesional, debe según el artículo 15,

Realizar un diagnóstico de situación entre los sujetos involucrados. El mismo será elaborado en forma interdisciplinaria y tendrá como objeto determinar los daños físicos o psíquicos sufridos por la víctima, evaluar la situación de peligro o riesgo y el entorno social (ley $\mathrm{N}^{\circ} 17.514$ ).

En función de lo planteado para analizar el dispositivo pericial partimos del análisis de los repertorios interpretativos (Wetherell y Potter, 1988) utilizados por los/as operadores/as judiciales para justificar y argumentar sus intervenciones. Por medio de la identificación de los repertorios empirista y profesionalizador visualizamos las estrategias de argumentación utilizadas para justificar las diferentes acciones así como también los posibles efectos o consecuencias sobre la judicialización de la VDC y el tratamiento que reciben las mujeres que transitan por el J LFE.

En este contexto, partimos de la noción de violencia como producto de ciertas estructuras elementales que a nivel socio cultural la sustentan y reproducen (Segato, 2003). Una de ellas es el género, en tanto parte fundamental de las relaciones sociales basadas en la diferencia sexual, pero fundamentalmente como forma de establecer relaciones de poder y subordinación generando de esta forma la construcción de vínculos jerárquicos (Scott, 1996). El género produce identidades, formas de ser y estar así como maneras de relacionarse constreñidas a determinadas normativas reguladoras que se transmiten con carácter de habitualidad y generan procesos de naturalización. Estos procesos de naturalización construyen identidades, producen y reproducen desigualdades, invisibilizan las diferencias y posibilidades, construyendo sujetos homogeneizados así como contribuye al orden heterosexual como única opción viable o permitida.

Considerar el género como una forma de hacer, una actividad incesante performada, en parte, sin saberlo y sin la propia voluntad, no implica que sea una actividad automática o mecánica. Por el

todo lo relativo a las pensiones alimenticias y, en su caso, a la guarda, tenencia y visitas (Ley № 17.514, artículo 10). contrario, es una práctica de improvisación en un escenario constrictivo. Además el género propio no se "hace" en soledad. Siempre se está "haciendo" con otro o para otro, aunque el otro sea sólo imaginario. (Butler, 2004/2006, p. 13).

Seguimos los planteos de J udith Butler en tanto postula al género como una producción performativa que impone prácticas reguladoras a partir de los efectos que produce (1990/2007) y entendemos que las prácticas judiciales y las periciales específicamente, operan como tecnologías de género (de Laurentis, 1989) a través de la fijación de sentidos inmutables y cuerpos generizados.

\section{Consideraciones metodológicas. Los aportes de la perspectiva etnográfica para el campo jurídico en cuestiones de Violencia Doméstica Conyugal}

El presente proyecto de investigación se construye en el marco de la Maestría en Psicología Social $^{2}$. El trabajo de campo es desarrollado por la maestranda y lo enmarcamos en los diseños teórico-metodológicos que responden a las perspectivas cualitativas de investigación social (Denzin \& Lincoln, 2003) y de producción de conocimiento situado (Haraway, 1995). Se trata de un estudio con enfoque etnográfico, en el sentido que plantea Rosana Guber (2001) de tener en cuenta la triple acepción de la etnografía en tanto su enfoque, método y texto. "Como enfoque la etnografía es una concepción y práctica de conocimiento que busca comprender los fenómenos sociales desde la perspectiva de sus miembros (entendidos como "actores", "agentes" 0 "sujetos sociales")" (Guber, 2001, p. 12).

Utilizamos como antecedentes varios estudios realizados en espacios jurídicos en diferentes contextos (Bodelón, 2012; Brasilinio, 2010; Cubells, 2002; Cubells, Albertín y Calsamiglia, 2010; Cubells, Calsamiglia y Albertín, 2009; Grabino, 2009). Cabe destacar la perspectiva planteada por J enny Cubells (2002) respecto a la etnografía jurídica cuyo objetivo principal es la problematización de la noción de justicia, a través de la pregunta por los valores que sustentan las prácticas jurídicas en tanto necesidad de objetividad e imparcialidad en su accionar.

\footnotetext{
2 Maestría en Psicología Social. Facultad de Psicología, Universidad de la República. Montevideo, Uruguay.
} 
La perspectiva etnográfica es postulada como un método singular dentro de las metodologías cualitativas de investigación social (Guber, 2001; Hammersley y Atkinson, 1983/ 1994). A punto de partida de este trabajo tomaremos esa singularidad-especificidad a modo de guía para el camino de la investigación. Seguiremos los principios que orientan esta perspectiva: reflexividad en tanto que posición ético-política desde donde posicionar la mirada (Pillow, 2003), flexibilidad en el trabajo de campo, presencia prolongada en el contexto y observación participante u observación etnográfica. Dice Clifford Geertz (2003):

Hacer etnografía es como tratar de leer (en el sentido de "interpretar un texto") un manuscrito extranjero, borroso, plagado de elipsis, de incoherencias, de sospechosas enmiendas y de comentarios tendenciosos y además escrito, no en las grafías convencionales de representación sonora, sino en ejemplos volátiles de conducta modelada (p. 24)

En este sentido, concurrimos a dicho escenario durante un lapso de cinco meses, entre julio y diciembre de 2013. Utilizamos un diseño flexible que permitió realizar modificaciones en el proceso de desarrollo de la estrategia de investigación. Para leer las perspectivas de los agentes nos nutrimos de los escenarios, los grupos, las personas y sus contextos, en función de esto la estrategia es situada, siendo todas las perspectivas valiosas. El eje de coherencia esta dado en función de dos elementos. Por un lado, por los objetivos de la investigación en torno al análisis de las narrativas construidas para la disposición de medidas de protección judicial en casos de VDC hacia la mujer, por parte de los/as operadores/ as de justicia de del JLEF. Y por otro lado, por la perspectiva teórica seleccionada a partir del análisis de la producción narrativa. Los trabajos sobre narrativas se comprenden en el campo de estudios del discurso y del lenguaje (Brockmeier y Harré, 2003; van Dijk, 1997).

Pensamos a las narrativas como una de las formas posibles de expresión por medio del lenguaje. A través de las mismas se configuran y se integran espacios de inteligibilidad, de comprensión inteligible del mundo, de construcción de sentidos y significados. Paul Ricoeur (1983; 1984) postula a la narración como la manera en que las personas se reconocen a sí mismas, transitan las experiencias y pueden dar cuenta de ellas (Lloret i Ayter, 2004). La potencialidad puesta en la construcción narrativa desde lo colectivo, es decir, las narrativas no son sólo producciones individuales, sino que al ser negociados los sentidos desde lo social, estas producciones son también colectivas (Gergen, 2007).

Las técnicas de producción de información que componen el corpus de análisis son: 10 entrevistas en profundidad a operadores/as judiciales de los JLEF, relevamiento y sistematización de 10 expedientes judiciales archivados, observación etnográfica y registro en diario de campo. El registro se caracteriza por recoger descripciones detalladas de las observaciones. Seguimos las sugerencias de Patton (1987/2002) y Cubells (2002), quienes especifican a las notas de campo como, descriptivas, compuestas por lo que dicen las personas implicadas, percepciones sobre los significados de las diversas situaciones, interpretaciones, vivencias y reflexiones sobre el análisis de las observaciones.

Por medio de estas técnicas nos acercamos a los sentidos que se construyen para la práctica pericial 'especializada en VDC' hacia la mujer.

\section{La inclusión de voces expertas}

Las voces expertas se integran al proceso judicial y constituyen un mojón de fundamental relevancia. Estas voces están dotadas de un posicionamiento particular, el lugar de la experticia profesional que le otorga la pertenencia a un determinado campo de conocimiento. Seguimos la idea de experticia a punto de partida de Nikolas Rose (1998) quien la relaciona con la atribución de ciertos entrenamientos y credenciales que hacen que algunas personas tengan una competencia especial. Competencias que generan lugares de autoridad y legitimidad a partir del manejo de un determinado saber.

Los dispositivos periciales se integran por diferentes disciplinas que implican recortes temáticos. En el espacio jurídico del JLEF se produce una fusión de algunos campos de conocimiento: disciplinas psi, medicina y trabajo social a los saberes jurídicos. Esta fusión se crea a partir del intercambio entre las disciplinas y de la existencia de un espacio y objetivos comunes. 


\begin{tabular}{|c|c|c|}
\hline \multirow{2}{*}{\multicolumn{3}{|c|}{$\begin{array}{c}\text { Entrevistas en profundidad } \\
\text { Operadores/as J udiciales y Equipo Técnico }\end{array}$}} \\
\hline & & \\
\hline \multicolumn{2}{|c|}{ Juez/a } & 2 \\
\hline \multicolumn{2}{|c|}{ Fiscal } & 1 \\
\hline \multicolumn{2}{|c|}{ Abogado/a Defensor/a } & 2 \\
\hline \multicolumn{2}{|c|}{ Psicología } & 2 \\
\hline \multicolumn{2}{|c|}{ Trabajo Social } & 1 \\
\hline \multicolumn{2}{|c|}{ Medicina Forense } & 1 \\
\hline \multicolumn{2}{|c|}{ Psiquiatría } & 1 \\
\hline \multicolumn{3}{|c|}{ Expedientes judiciales } \\
\hline$\underset{\mathbf{N}^{\circ}}{\operatorname{Exp}}$ & \multicolumn{2}{|c|}{ Contenido de Expediente } \\
\hline 1 & \multicolumn{2}{|c|}{$\begin{array}{l}\text { Partes policiales, } 4 \text { Audiencias, } 5 \text { Escritos, } \\
2 \text { Informes periciales }\end{array}$} \\
\hline 2 & \multicolumn{2}{|c|}{$\begin{array}{l}\text { Partes policiales, Parte médico, } 3 \text { Audiencias, } \\
2 \text { Informes periciales }\end{array}$} \\
\hline 3 & \multicolumn{2}{|c|}{$\begin{array}{l}\text { Partes policiales, Partes médicos, } 2 \text { Audiencias, } \\
3 \text { Informes periciales, } 1 \text { Escrito }\end{array}$} \\
\hline 4 & \multicolumn{2}{|c|}{3 Escritos, 3 Audiencias, 2 Informes periciales } \\
\hline 5 & \multicolumn{2}{|c|}{$\begin{array}{l}\text { Partes policiales, } 3 \text { Audiencias, } \\
\text { 1 Informe pericial }\end{array}$} \\
\hline 6 & \multicolumn{2}{|c|}{$\begin{array}{l}\text { Partes policiales, } 2 \text { Audiencias, } \\
2 \text { Informes periciales }\end{array}$} \\
\hline 7 & \multicolumn{2}{|c|}{$\begin{array}{l}\text { Partes policiales, } 2 \text { Audiencias, } \\
1 \text { Informe pericial }\end{array}$} \\
\hline 8 & \multicolumn{2}{|c|}{$\begin{array}{l}\text { Partes policiales, Pruebas (imágenes de } \\
\text { mensajes de texto de celular y mails), } 2 \\
\text { Escritos, } 2 \text { Audiencias, } 1 \text { Informe pericial }\end{array}$} \\
\hline 9 & \multicolumn{2}{|c|}{$\begin{array}{l}\text { Partes policiales, } 3 \text { Audiencias, } \\
2 \text { Informes periciales }\end{array}$} \\
\hline 10 & \multicolumn{2}{|c|}{$\begin{array}{l}\text { Partes policiales, } 2 \text { Audiencias, } \\
2 \text { Informes periciales }\end{array}$} \\
\hline
\end{tabular}

\section{Observación etnográfica}

Lugares donde se realizan observaciones

Oficina de la sede del J uzgado

Sala de espera de denunciantes

Sala de espera de denunciados/as

Sala de audiencias

Pasillos

Alrededores del J uzgado

Tabla 1: Corpus de análisis
Nos interesa conocer el funcionamiento que el dispositivo pericial realiza en el campo específico de la VDC ejercida hacia las mujeres. Siguiendo las reflexiones sobre la concepción de experticia interrogamos al dispositivo pericial y su lugar en la construcción de las narrativas de los/ as operadores/ as de justicia para la disposición de las medidas de protección judicial en las situaciones de VDC en los J LFE. A través de esta apuesta nos vamos a detener en dos niveles de análisis, por un lado aquel que da cuenta de la construcción de la verdad a través de las cualidades jurídicas y por otro la evaluación de la personalidad como un elemento central en la actividad pericial.

Para dar andamiaje a estas reflexiones nos apoyamos en el análisis de los repertorios interpretativos (Wetherell y Potter, 1988) como "elementos esenciales que los hablantes utilizan para construir versiones de las acciones, los procesos cognitivos y otros fenómenos" (Wetherell y Potter, 1988, p. 3). Los mismos son un insumo principal en la construcción de las versiones de los acontecimientos, se utilizan para justificar versiones, anticipar el discurso del otro/a y producir la credibilidad (Cubells, 2002). Se identifican dos repertorios interpretativos dentro de la comunidad jurídica: el repertorio empirista (Gilbert $\&$ Mulkay, 1984; Potter, 1998) y el repertorio profesionalizador (Cubells, 2002). Ambos se encuentran relacionados y los utilizamos como herramientas de análisis para problematizar el funcionamiento del dispositivo pericial especializado en VDC.

\section{La construcción de la verdad a través de las cualidades jurídicas}

A partir de esta relación de convivencia, estos campos de conocimiento que no provienen de lo jurídico estrictamente, realizan un proceso de acomodamiento de sus prácticas profesionales a los fines jurídicos, donde aquello que denominamos cualidades jurídicas cobra un papel clave. Llamamos cualidades a ciertos aspectos que describen y hacen a la especificidad de la tarea judicial y que se ponen en juego en el desarrollo de las tareas que integran al proceso. Dentro de estas cualidades que persiguen como objetivo principal la construcción de la verdad a partir de los hechos denunciados hallamos: objetividad, imparcialidad, búsqueda de la verdad/pruebas, neutralidad (Bodelón, 2012; Cubells, 2002). 
La capacidad de desarrollar estas aptitudes va signado las tareas que desarrollan los/ as diferentes operadores/ as jurídicos así como también quienes integran el cuerpo pericial. Consideramos que para realizar este movimiento de acomodamiento y su consiguiente legitimación, las disciplinas extra jurídicas intentan también poder dar cuenta de éstas aptitudes, a la vez que, a través del estatus científico, otorgan legitimidad a los objetivos jurídicos y sus pretendidas cualidades. En este sentido los informes periciales constituyen piezas clave para el análisis de estas cualidades. Entendemos a los mismos como aquellos que expresan enunciados judiciales privilegiados en el entendido de Foucault (1999), dado que ocupan un lugar especial en la construcción de la verdad jurídica y expresan un valor de cientificidad.

Para el análisis de los informes periciales, recurrimos al repertorio empirista (Gilbert \& Mulkay, 1984; Potter, 1998) presente en el discurso de los/as operadores/as de justicia y en los informes periciales para comunicar y describir las acciones o transmitir las decisiones judiciales. El repertorio empirista se caracteriza en su uso por mostrar los datos, en este caso, los hechos desde un lugar de centralidad. Es decir, los datos respecto a los hechos de violencia o los vínculos de VDC ocupan un lugar central y se muestran con capacidad de agencia. De esta manera se identifica el uso de este repertorio mediante la observación de tres características; impersonalidad gramatical, preponderancia de los datos y uso de reglas universales (Potter, 1998).

El uso de este repertorio tiene la función de generar la idea de objetividad e imparcialidad, desconociendo la incidencia de los procesos de interpretación por parte de los/as operadores/as que pueden estar influyendo en la transmisión de la información así como la influencia del contexto de producción. Podemos ver cómo la actividad pericial colabora como una herramienta más en la elaboración de versiones de los hechos, por medio del uso del repertorio empirista visualizamos el carácter impersonal con el cual en las pericias se transmiten los hechos o los datos y cómo estos de alguna manera aparecen como ajenos a las personas mismas. J unto con los efectos de imparcialidad y objetividad que generan, observamos que la "institución presenta los hechos como externos describiéndolos co- mo si éstos tuvieran lugar con independencia a la práctica jurídica" (Cubells, 2002, p. 67).

Vemos como algunas prácticas periciales permiten analizar el uso del repertorio empirista y de una retórica que busca transmitir la existencia o no de la veracidad del relato. Sí bien el objetivo de la actividad pericial no es el de servir en calidad de prueba. Esta función aparece solapada en los mismos bajo una estrategia discursiva en el plano de las impresiones o de las percepciones del ETEC.

\begin{abstract}
Ratifica su denuncia y lo hace de un modo consistente, por lo que su relato provoca la impresión de que es verosímil (Expediente №4, material de investigación).

Debe indicarse que el relato de la denunciante impresiona como absolutamente veraz y consistente. Basta leer algunos de los mensajes del denunciado para observar la virulencia que estos contienen. Puede interpretarse que estos textos son la expresión de un hombre que se siente hondamente herido en su amor propio (Expediente №8, material de investigación).
\end{abstract}

En estos ejemplos citados de las pericias podemos avizorar cómo la estrategia discursiva es utilizada con rasgos de ambigüedad. Es decir, dado que no es parte de sus cometidos afirmar o negar de forma tajante los datos recabados, se realizan los planteos en términos de sensaciones. Pero estas sensaciones 0 percepciones del ETEC se encuentran fundadas en elementos consistentes, en interpretaciones que se afirman. Así se conjuga la información que se quiere transmitir; el contenido, con la forma de transmitirla; la estrategia. Los efectos buscados se relacionan con la producción de verdad y la legitimación de la intervención judicial.

A su vez indagan respecto al cumplimiento 0 no de las medidas de protección dispuestas. Por ende la actividad pericial vinculada a los hechos cumple la función de comprobar los mismos, contraponer versiones, monitorear el cumplimiento de las medidas y se suma a esta función de prueba, la opinión experta respecto a la apariencia de verdad en los relatos. Esta opinión respecto a la veracidad de los relatos, se transforma en prueba gracias a la validez, eficacia y autenticidad atribuida a los discursos expertos. Al mismo tiempo visualizamos las relaciones entre la búsqueda de verdad y la búsqueda de justicia garantizando las mismas por medio del recurso a lo científico. Se afirma la noción de "verdad y justicia como una ecuación de igualdad" (López Ga- 
llego, 2010, p. 400). En referencia a estas ideas situamos de los informes,

Minimiza el conflicto de pareja. No niega la posibilidad de reiniciar el vínculo con la denunciante y afirma estar cumpliendo las medidas cautelares (Expediente № 9, material de investigación).

Con respecto a la denuncia ofrecen versiones contrapuestas: la denunciante la confirma en todos sus términos, en tanto que el denunciado la desestima. El denunciado desestima la denuncia en tanto que solo admite discusiones e insultos recíprocos (Expediente № 8, material de investigación).

Por otro lado y a partir de basarse en todo aquello relativo a lo fáctico, se realiza un análisis por parte del ETEC que intenta predecir o anticipar la posibilidad de que se vuelvan a producir nuevos hechos de violencia. Para realizar estas predicciones utilizan como insumo los mismos hechos relatados y algunas características que podrían ser propiciadoras de esta repetición. La estrategia retórica que utilizan para validar la argumentación se vincula a dos elementos. Por un lado la descripción de la personalidad de los/ as involucrados/ as, otorgando un lugar central a la valoración de la proximidad (geográfica 0 afectiva) de las personas así como a la comunicación que establecen. Estas valoraciones respecto a la forma de vincularse se encuentran además adheridas a las disposiciones de la Ley № 17.514. Un segundo elemento de la estrategia tiene que ver con el uso estereotipado que se hace respecto a la forma de relacionamiento entre denunciado y denunciante. De esta manera toman como base los modelos tradicionales de expresión de la VDC para intentar predecir los hechos futuros o una reiteración de algún hecho de violencia.

Se considera que hasta la fecha no se han vuelto a suscitar situaciones de violencia, no descartándose la posibilidad de reiteración de nuevos episodios, ante el hecho de que la pareja permanece residiendo en el mismo predio, persistiendo un clima tenso de convivencia (Expediente №10, material de investigación).

De mantenerse contacto entre las partes se reiterarían episodios similares (Expediente № 4, material de investigación)

Es altamente probable que se reiteren situaciones similares a la de autos de reiniciarse la convivencia y en grado creciente de violencia (Expediente № 9, material de investigación).

Por las características de uno y otro de los entrevistados impresiona tratarse de una situación de riesgo potencial en el sentido de que pueden repetirse graves hechos como los denunciados (Expediente № 2, material de investigación).
Visualizamos en estos ejemplos como la actividad pericial aparece ceñida a la aplicación ritual de la ley de VD, resaltando en los informes aspectos relativos a las medidas de protección, principalmente a las medidas de acercamiento y comunicación. De esta manera los informes periciales cumplen con controlar y dar seguimiento a la verificación del cumplimiento de medidas, en lugar de poder brindar consideraciones o contribuciones relativas a su especificidad disciplinar.

\section{La evaluación de la personalidad: un elemento central en la actividad pericial}

La conceptualización que construyen sobre la VDC, así como sobre las personas implicadas en ese proceso, muestra la influencia del síndrome de la mujer maltratada, el ciclo de la violencia (Walker, 1979/2012) y el modelo ecológico (Bronfenbrenner, 1987). Estos modelos sobre la noción de violencia construyen marcos de intervención, un tipo de situación y un tipo de mujer específico que contiene ciertas características. Esta conceptualización ha construido ideas estereotipadas de mujer que se convierten en lo esperado para la determinación de VDC, dejando de lado otras mujeres con otras particularidades. Es decir, los/as operadores/as del J LEF esperan a una mujer con determinadas características: sumisa, dañada, con años de relación de pareja violenta, baja autoestima, entre otras. Estas características se relacionan frecuentemente con aquellas situaciones en las cuales la violencia se ha venido sosteniendo durante muchos años. Conjuntamente el J LEF ha estado recibiendo situaciones que responden a otras características y que por algún motivo están pudiendo llegar ahora y no antes. Este hecho hace que los/ as operadores/ as se encuentren con mujeres que tienen relatos e historias de vida muy diversos. La dificultad versa sobre la posibilidad de ver, escuchar y percibir elementos que no se corresponden con lo que ellos denominan "la violencia clásica o violencia común" (Registro de diario de Campo, 29 de octubre de 2013) como lo llaman frecuentemente. Esta dificultad impide comprender la diversidad de las historias de vida que puedan llegar, las necesidades y mucho más las expectativas de los/ as usuarios/ as.

Acá la mayoría de casos que llegan no son violencia, violencia. Son conflictos de la pareja, hostilidades. Se pegan ambos, vos preguntas y él te dice ella me pegó primero y te muestra la marca, 
la violencia típica es la que menos se ve ( $\mathrm{uez} / \mathrm{a}$, comunicación personal, 31 de octubre de 2013$)^{3}$.

Desde los más de diez años que viene trabajando el JLEF se han ido edificando algunas concepciones que se sustentan básicamente en la repetición o en la frecuencia con la cual observan ciertas características. Es decir, la fundamentación de la intervención es a partir de la experiencia de trabajo. Sin embargo esa frecuencia o reiteración de hechos no son insumos fiables para una conceptualización sino que por el contrario obstaculizan la posibilidad de comprender y analizar las diferentes situaciones que atienden.

Visualizamos el uso del repertorio profesionalizador en el accionar judicial y también en las prácticas periciales. Este repertorio es aquel por medio del cual se visualiza cómo el saber técnico y pragmático de las diferentes disciplinas que se ocupan de la actividad pericial tienen determinada preponderancia sobre el saber teórico o aquel que lleva adelante mayores niveles de análisis (Cubells et al., 2010). El repertorio profesionalizador se identifica a partir de la relevancia otorgada a la experiencia y a la práctica profesional que construye especificidades disciplinares y genera profesionales especializados en algunas áreas, en este caso en VDC.

Por otro lado identificamos el uso de este repertorio en la utilización de protocolos de intervención, guías y normativas que se van colocando por encima de la posibilidad de reflexionar y/ o cuestionar las prácticas profesionales cotidianas. Se visualiza en los informes periciales una centralidad a la hora de describir las características de personalidad. En general esta descripción es realizada por las disciplinas psi (Rose, 1998). Se lleva a cabo un cierto destaque de estas características sin brindar elementos que permitan la comprensión de esos aspectos destacados lo cual deja entrever esta argumentación a partir de la validez que otorga la especialización en la temática.

Además se relaciona con los análisis realizados por Foucault (1999) donde muestra cómo a través de las pericias se transita desde los hechos a la conducta y a la manera de ser. De alguna manera por medio de este énfasis en

\footnotetext{
${ }^{3}$ Los nombres son omitidos para proteger aspectos de confidencialidad en términos de identidad de los/ as participantes.
}

los rasgos de personalidad que se destacan se intenta mostrar una forma general de la conducta de las personas que las hace proclives a establecer vínculos violentos. Además se construye la personalidad en "términos de individualidad, interioridad, totalidad, unicidad y estabilidad. (...) se habilita la posibilidad de control (...) debido a que se pueden pronosticar comportamientos futuros e inferir los pasados, en función de las características de personalidad de los sujetos involucrados" (López Gallego, 2010, pp. 401-402).

Esta construcción de la personalidad por medio de la evaluación experta permite dejar plasmado gracias a la pericia aquello que puede ser invisible a los ojos de los/as jueces/ as, pero no a la mirada experta. Así como también permite categorizar a las personas a través de lo que son y no por medio de lo que hacen (Rose, 1989/ 1999).

\begin{abstract}
Su nivel intelectual es normal, no presenta signos de patología psiquiátrica descompensada. Sus características de personalidad favorecen los conflictos vinculares y conductas violentas por lo que se beneficiaría de mantener tratamiento psicopsiquiátrico (Expediente № 10, material de investigación).
\end{abstract}

Sus rasgos de personalidad favorecieron el inicio del vínculo con la conflictiva vincular, inestabilidad afectiva de la denunciante (Expediente № 4, material de investigación).

Podemos observar en estos ejemplos el uso del saber profesional fundamentado antes que nada en la experiencia y la casuística. Estos hechos funcionan como argumentos para establecer explicaciones, realizar predicciones y estimaciones respecto al pronóstico de las relaciones de pareja. La utilización de este repertorio permite la inclusión de estos elementos de especulación respecto a la relación de pareja en las decisiones que se toman en el proceso judicial. Este hecho tiene efectos en la construcción de una relación de poder asimétrica entre expertos y usuarios/as, desterrando e invisibilizando las voces y opiniones de los/as protagonistas. La especialización en la temática de VDC brinda una credencial al ETEC que enmascara el uso de prejuicios y estereotipos dominantes en la intervención judicial.

En el contexto de la intervención judicial especializada en VDC identificamos el uso de los repertorios interpretativos empirista y profesionalizador en diferentes momentos de la actividad pericial. Las distintas estrategias retó- 
ricas utilizadas por el ETEC muestran una finalidad de contribuir a la legitimación de la veracidad y prueba de los hechos o realizar una evaluación de la personalidad de los/as involucrados/ as.

Sin bien en el proceso de la investigación no reaunimos las voces de las protagonistas de primera mano. Podemos inferir en posibles efectos negativos o contraproducentes de la intervención judicial.

Uno de los efectos posibles es la tendencia a la homogeneización y universalización de la diferencia de las historias de vida. La intervención realizada a partir de cierta naturalización genera la idea de un solo tipo esperable de situación de VDC.

Como otro efecto contingente, observamos procesos de individualización por medio de la fragmentación de las historias de vida y de las historias de violencia. Se produce un acceso parcial a los problemas planteados por las mujeres a partir del intento de adecuar lo que le sucede a las personas a la legislación 0 a las condiciones de la intervención. Se descontextualizan así las historias de vida para traducirlas en problemas jurídicos.

\section{Reflexiones finales. J udicialización de la intimidad y las relaciones cotidianas en el marco del saber experto}

Pedir ayuda al sistema de justicia cuando existe un problema entre las personas es una estrategia posible entre muchas otras. El acceso al sistema de justicia da cuenta de un enorme ámbito de intervención con características muy particulares según el área de especialización. Nos detendremos aquí en la esfera de la judicialización, como una de las tantas esferas que intervienen sobre la organización de la vida social, de la vida en familia y la vida en relaciones de pareja especialmente por medio del saber experto.

El derecho, a partir de la intromisión en los avatares familiares ha ido ganando terreno en la participación de la organización social, haciéndose cada vez más presente y necesario para el establecimiento de un orden y el cumplimiento de algunas normas. Tomamos la noción de judicialización de Theópilos Rifiotis (2004) quien plantea que dichos procesos "traducen un doble movimiento: de un lado la ampliación de acceso al sistema judiciario y por otro, la desvalorización de otras formas de resolución del conflicto" (p. 114). El autor trabaja este concepto en el ámbito de las relaciones sociales, de la vida íntima de las personas. Así señala que la judicialización de la vida cotidiana genera entre otros efectos, efectos de violentación.

Valoramos a la intervención judicial como una acción performativa que reactualiza las representaciones que dan forma y constituyen el problema. Butler (1993/2005) plantea que los actos performativos se dan a partir de la reiteración, de la persistencia, pero no de cualquier forma de repetir sino de aquella que sigue rituales en determinados contextos. Reflexionamos que la intervención judicial, en especial la realizada por los equipos técnicos, en la vida privada de las personas es una acción que mediatiza los vínculos, que introduce terceros como escenario de representación del conflicto, agregando voces, opiniones y miradas que dan forma al problema en cuestión. Estas voces generan efectos y producen diversos sentidos.

El dispositivo pericial en el campo de la VDC construye determinadas versiones acerca de algunas nociones como familia, mujer, víctima, violencia y personalidad. Los/as técnicos/ as que realizan la actividad pericial provienen de disciplinas ajenas a las ciencias jurídicas y por medio del proceso de construcción de estas versiones producen un movimiento de acercamiento a las expectativas y necesidades jurídicas. De esta manera estos saberes expertos comienzan a reproducir en el ritual de intervención judicial aquellas cualidades que conforman los procesos jurídicos, por medio de la búsqueda de verdad, en un accionar que se caracteriza por ser objetivo e imparcial.

El desarrollo de las funciones periciales colabora con la necesidad de construcción de pruebas para confirmar y afirmar los datos que traen al J LEF los/ as implicados en el proceso. A su vez por medio de esta actividad también brindan elementos que contribuyen en la legitimación de ciertos estereotipos vinculados a las parejas que viven situaciones de violencia. De esta manera el saber experto y la especialización en la temática de violencia, como sello distintivo, legitiman el accionar jurídico plagado del uso de prejuicios y estereotipos. 
Finalmente, en el dispositivo pericial la personalidad es construida en términos de individualidad, interioridad, unicidad y estabilidad. A partir de estas características, se habilita la posibilidad de pronóstico y control de las personas involucradas, debido a que se pueden predecir comportamientos futuros e inferir los pasados. Cubells (2002) plantea que una de las estrategias de los agentes jurídicos es el presupuesto de unicidad entre el acto y el actor. La naturaleza del acto dependerá de la naturaleza del actor, es decir de la persona involucrada. Se naturaliza así el concepto de personalidad convirtiéndolo en una verdad objetiva, que habilitará prácticas de gobierno donde el dispositivo pericial opera descifrando los códigos de la personalidad de los actores involucrados.

Las estrategias de judicialización y las intervenciones de los saberes expertos al decir de Rifiotis (2004) desestiman otras formas posibles de enfrentar los conflictos que se pueden dar en otros planos como lo familiar o lo comunitario. Las tensiones que subyacen 10 planteado se imbrican en las ideas que vinculan la posibilidad de cambio, de movimiento, de transformación. Palabras que se conectan con la concepción de agencia y con el deseo de recuperar la posibilidad de que el acceso a la justicia se constituya en una experiencia de restitución de derechos y apertura de nuevos horizontes y no en una experiencia de devastación de lo subjetivo y corrimiento del lugar de los/ as protagonistas.

La apuesta es contribuir a producir un ámbito de respeto y cuidado que logre brindar respuestas y generar oportunidades preservando sobre todo las decisiones e intereses de los/ as involucrados/as. La intención es recuperar las dimensiones socio históricas, políticas y éticas para producir cuestionamientos e interrogantes. El desafío para la construcción de una práctica pericial situada queda planteado.

\section{Referencias}

Bodelón, Encarna (2012). Violencia de género: y las respuestas de los sistemas penales. Buenos Aires: Ediciones Didot.

Brasilinio, Jullyane (2010). Masculinidades no juizado de violência doméstica e familiar contra a mulher: performances em cena. Tesis de Maestría sin publicar, Universidade Federal de Pernambuco.
Brockmeier, J ens \& Harré, Rom (2003). Narrativa: Problemas e Promesas de um Paradigma Alternativo. Psicologia: Reflexão e Critica, 16(3), 525-

535. http:// dx. doi. org/ 10.1590/ S010279722003000300011

Bronfenbrenner, Urie (1987). La ecología del desarrollo humano. Barcelona: Paidós.

Butler, J udith (1993/2005). Cuerpos que importan: sobre los límites materiales y discursivos del "sexo". Barcelona: Paidós.

Butler, J udith (2004/ 2006). Deshacer el género. Barcelona: Paidós.

Butler, J udith (1990/ 2007). El género en disputa: el feminismo y la subversión de la identidad. Barcelona: Paidós.

Cubells, J enny (2002). Construcción social del delito: un estudio etnográfico en la práctica del derecho penal. Tesis de Doctorado sin publicar, Universitat Autónoma de Barcelona.

Cubells, J enny; Albertín, Pilar \& Calsamiglia, Andrea (2010). Transitando por los espacios jurídico-penales: discursos sociales e implicaciones para la intervención en casos de violencia hacia la mujer. Acciones e investigaciones sociales, 28, 79-108. Recuperado de

https: / / papiro. unizar.es/ ojs/ index. php/ ais/artic le/ view/ 482

Cubells, J enny; Calsamiglia, Andrea \& Albertín, Pilar (2009). El ejercicio profesional en el abordaje de la violencia de género en el ámbito jurídicopenal: un análisis psicosocial. Anales de Psicología, 26(1), 369-377. Recuperado de http:// www.um. es/ analesps/ v26/ v26 2/ 22$\underline{262 . p d f}$

de Laurentis, Teresa (1989). Technologies of Gender. Essays on Theory, Film and Fiction. Londres: Macmillan Press.

Deleuze, Gilles (1989/1999). ¿Qué es un dispositivo? En Etienne Balibar, Gilles Deleuze, Hubert Dreyfus, Frank Manfred, André Glücksmann, Gérard Lebrun, Roberto Machado... J aques Alain Miller (Eds.), Michel Foucault, filósofo (pp. 155163). Barcelona: Gedisa.

Denzin, Norman \& Lincoln, Yvonna (Eds.) (2003). The Landscape of Qualitative Research. Theories and Issues. Thousand Oaks: Sage.

Foucault, Michel (1999). Los anormales. Buenos Aires: Fondo de Cultura Económica.

Geertz, Clifford (2003). La interpretación de las culturas. Barcelona: Gedisa.

Gergen, Kenneth (2007). Construccionismo social, aportes para el debate y la práctica. Bogotá: Universidad de Los Andes. 
Gilbert, Nigel \& Mulkay, Michael (1984). Opening Pandora's box: a Sociologj cal Analysis of Seientist's discourse. Camhtidge: Cambridge University Press.

Grabino, Valeria (2009). Estudio de las limitaciones materiales, culturales y de formación de los/ as operadores/ as del Poder J udicial para la implementación de la Ley № 17.514, en la actualidad. En Susana Rostagnol (Coord.), No era un gran amor: 4 investigaciones sobre violencia doméstica (pp. 67-124). Montevideo: INMUJ ERES/ MIDES.

Guber, Rosana (2001). La etnografía, método, campo y reflexividad. Bogotá: Grupo Editorial Norma.

Hammersley, Martyn \& Atkinson, Paul (1983/ 1994). Etnografía: métodos de investigación. Barcelona: Paidós.

Haraway, Donna (1995). Ciencia, cyborgs y mujeres. La invención de la naturaleza. Madrid: Cátedra.

Ley № 15.982 Código General del Proceso. Recuperado de: http:/ / www.parlamento.gub.uy/ leyes/ AccesoTe xtoLey. asp?Ley $=15982 \&$ Anchor $=$

Ley № 17.514 Violencia Doméstica (D. 0.9 jul/ 002 - № 26045) Recuperado de: http:// www. parlamento. gub.uy/ leyes/ AccesoTe xtoLey. asp?Ley $=17514 \&$ Anchor $=$

Lloret i Ayter, Imma (2004). Enfoque narrativo y violencia: intervención con mujeres que sufren maltrato. Revista Intervención psicosocial. 13(2), 165-175. Recuperado de http:/ / www.copmadrid.org/ webcopm/ publicaci ones/ social/ 91389.pdf

López Gallego, Laura (2010). Una apuesta analítica del funcionamiento del dispositivo psi pericial en el campo penal. Psicología \& Sociedade, 22(2), 396-404. http:/ / dx. doi.org/ 10.1590/ S0102$\underline{71822010000200021}$

Pacheco, Luis (2013). El proceso de violencia doméstica: aspectos civiles y penales de la violencia doméstica en el Uruguay. Montevideo: AMF.

Patton, Michael (1987/ 2002). Qualitative research $\&$ evaluation methods. London: Sage Publications.

Pillow, Wanda (2003). Confession, catharsis, or cure? Rethinking the uses of reflexivity as meth- odological power in qualitative research. Qualitative Studies in Education, 16(2), 175-196. http:/ / dx. doi.org/ 10.1080/095183903200006063 $\underline{5}$

Potter, J onathan (1998). La representación dela realidad Discurso, retórica y construcción social. Barcelona: Paidós.

Ricoeur, Paul (1983). Tiempo y narración I Configuración del tiempo en el relato histórico. Madrid: Cristiandad.

Ricoeur, Paul (1984) Tiempo y narración II Configuración del tiempo en el relato de ficción. Madrid: Cristiandad.

Rifiotis, Theofilos (2004) As delegacias especiais de proteçao à mulher no Brasil e a «J udiciarizaçao» dos conflitos conjugais. Sociedade e Estado, 19(1), 85- 119. http:/ / dx.doi. org/ 10.1590/ S0102$\underline{69922004000100005}$

Rose, Nikolas (1998). Inventing our selves: psychology, power and personhood. Cambridge: Cambridge University Press.

Rose, Nikolas (1989/ 1999). Governing the soul. The shaping of the private self. Free Association. London: Books.

Scott, J oan (1996). El género: una categoría útil para el análisis histórico. En Marta Lamas (Comp.), El género: la construcción cultural de la diferencia sexual (pp. 265-302). Ciudad de México: PUEG.

Segato, Rita (2003). Las estructuras elementales de la violencia. Ensayos sobre género entre la antropología, el psicoanálisis y los derechos humanos. Buenos Aires: Universidad Nacional de Quilmes.

van Dijk, Teun (1997). Discourse studies. London: Sage.

Walker, Leonore (1979/2012). El síndrome de la mujer maltratada. Bilbao: Desclée de Brower.

Wetherell, Margaret \& Potter, J onathan (1988). El análisis del discurso y la identificación de los repertorios interpretativos. Recuperado de: https: / / www.academia.edu/ 2601270/ El an\%C3\% Allisis del discurso y la identificaci $\% 3 \% 33 n$ d e los repertorios interpretativos 


\section{Cecilia Montes Maldonado}

Docente Ayudante del Programa de Psicología Social Comunitaria de la Facultad de Psicología, Universidad de la República. Magíster en Psicología Social por la Facultad de Psicología, Universidad de la República. Trabaja en temáticas de violencia de género y estudios de género, especialmente violencia doméstica y las relaciones con el ámbito jurídico.

\section{LAURA LÓPEZ GALLEGO}

Profesora Adjunta del Programa de Psicología Social Comunitaria de la Facultad de Psicología, Universidad de la República. Magíster en Psicología Social y Doctoranda de los Estudios Doctorales en Psicología Social de la Universidad Autónoma de Barcelona. Trabaja en temáticas que vinculan las prácticas psi, los sistemas penales y el género.

DIRECCIÓN DE CONTACTO

cmontes@psico.edu.uy

\section{FORMATO DE CITACIÓN}

Montes Maldonado, Cecilia \& López Gallego, Laura (2015). La experticia profesional en la judicialización de la violencia doméstica conyugal. Quaderns de Psicologia, 17(2), 7-18. http:// dx. doi.org/ 10.5565/ rev/ qpsicologia. 1237

\section{HISTORIA EDITORIAL}

Recibido: 16/ 10/2014

10 Revisión: 14/07/2015

2o Revisión: 02/ 10/2015

Aceptado: 17/ 10/2015 